Diagnostic methodology and treatment modalities for the 2year periods before (2007-8) and after (2010-11) the specialist MDT formation are given in the table (Table 1). There was no difference in histological rates $(100 \%$ and $92 \%$ respectively) or cell types between the two periods.

Conclusions In the second time period, our use of radical oncological treatment increased and at the same time extensive surgical treatment decreased, in keeping with current considered best practice. These changes in clinical practice coincided with discussion of these cases at the newly formed MCCN specialist mesothelioma MDT. This study emphasises the value of an expert multidisciplinary approach to the management of this unfortunate group of patients.

\section{P216 ANALYSING THE SUCCESS RATES OF PLEURODESIS IN PATIENTS ADMITTED TO AN ELECTIVE SHORT STAY WARD}

AB Bibi, SW Wilson, NA Anwar; Hospitals East Lancashire NHS Trust, Blackburn, England

10.1136/thoraxjnl-2013-204457.368

Introduction Pleural effusions are a common respiratory problem and account for 20,000 hospital episodes annually. $70 \%$ of patients need admission of 7 days or more (1). Treatment options include pleurodesis and long term indwelling pleural catheters (IPC)(2). Traditionally, management has involved recurrent lengthy hospital admissions which are both inconvenient for the patient and expensive. To reduce these problems, we admit patients to an elective short stay ward where they undergo pleurodesis and are discharged after 1-2 days.

Aim To assess the effectiveness of pleurodesis in patients admitted to an elective short stay ward.

Method We retrospectively reviewed 33 patients who were managed on an elective ward. A $12 \mathrm{~F}$ Chest drain was inserted followed by administration of $4 \mathrm{~g}$ sterile talc. Patients who did not re-attend with an effusion within in the following six months, or prior to dying were considered a success.

Results During 2009-2010, 33 patients with an average age of 66.5 years underwent pleurodesis on the elective short stay ward. 23/33 patients did not re-attend with an effusion within six months (70\%). However, 10 re-attended with a recurrent effusion despite talc (30\%). Of these, 3 patients had trapped lung on their xray and 1 had a chylothorax. Both are reported causes of failure (2).Chest drains stayed in for 1-2 days, with an average inpatient stay of 2 days. 18/33 patients died within 3 months of admission (54\%). Of the 10 that re-attended, 6 died within 3 months (60\%). There were no complications resulting from this procedure.

Conclusions Patients who are admitted to an elective short stay ward are managed safely and effectively. They require a shorter inpatient hospital stay which is cost-effective. Our results illustrate $70 \%$ of patients did not require an IPC but treatment with pleurodesis alone was sufficient to prevent re-attendances.Most patients do not require an acute hospital admission. Patients who do re-attend with a recurrent effusion due to trapped lung can be considered for IPC.

\section{REFERENCES}

1. Hospital statistics for pleural effusion. DoH.2002-2003. (Accessed June 2013) www.rightdiagnosis.com/p/pleural_effusion/stats.htm.

2. Roberts ME, Neville E et.al. BTS Pleural Disease Guideline. Management of a malignant pleural effusion: Thorax;2010;65(2)

\section{P217 DOES CHEMOTHERAPY INCREASE THE RISK OF DEVELOPING PLEURAL INFECTIONS IN PATIENTS WITH INDWELLING PLEURAL CATHETERS?}

H Edwards, L Bishop; Portsmouth Hospital Trust, Portsmouth, United Kingdom

10.1136/thoraxjnl-2013-204457.369

Introduction Indwelling Pleural Catheters (IPC) are indicated for the management of recurrent or resistant Malignant Pleural Effusions (MPE), usually after talc pleurodesis. While generally safe and effective, they carry a risk of pleural infection thought to be $4.7 \%[1]$. We have noticed a number of empyema's in patients receiving chemotherapy with an IPC, and want to see if there is an increased risk for IPC associated infection with chemotherapy in MPE above that in the general population.

Methods We reviewed all patients in our hospital who have received an indwelling pleural catheter, from the implementation of the service in February 2011 until June 2013. We reviewed patient details, indication for IPC, possible or definite infection post procedure and relation to chemotherapy. This information was obtained from patients' medical records, pathology reports and the radiology system.

Results 86 IPC's have been inserted from February 2011 until June 2013. Five of these were replacement drains (replaced due to blockage, displacement or infection). A total of 21/86 (24\%) patients had chemotherapy either immediately before IPC insertion or with IPC in place. 11 patients $(12.8 \%)$ were treated for suspected pleural infections, but only 5 patients $(5.8 \%)$ were confirmed with positive pleural cultures. 3 of the 5 patients were undergoing chemotherapy at the time of the infection

Conclusions Patients receiving indwelling pleural catheters are usually those with a malignant process and therefore chemotherapy is a common treatment used in this population. Although our numbers are small, they suggest that there may be an increased risk of pleural infection in patients with an IPC who undergo chemotherapy. Until a larger analysis can be done, it would be reasonable to consider prophylactic antibiotics during catheter insertion if a patient is due to have chemotherapy.

\section{REFERENCES}

1. Fysh ET et al. Clinical Outcomes of Indwelling Pleural Catheter-Related Pleural Infections: an international multicenter study. Chest. 2013 Jul 4

\begin{tabular}{lllll} 
Abstract P217 Table 1. & \\
\hline Patient & Indication & Cultures & $\begin{array}{l}\text { Timing of } \\
\text { infection }\end{array}$ & Chemotherapy \\
\hline 1 & Lung cancer & $\begin{array}{l}\text { S. aureus \& Group C } \\
\text { Strep from pleural fluid }\end{array}$ & $\begin{array}{l}\text { Time of } \\
\text { insertion }\end{array}$ & No \\
& Breast cancer & $\begin{array}{l}\text { Pseudomonas from } \\
\text { pleural fluid }\end{array}$ & 8 months & Yes \\
2 & Lung cancer & $\begin{array}{l}\text { S. aureus from pleural fluid } \\
3\end{array}$ & 3 weeks & No \\
4 & Mesothelioma & S. aureus from pleural fluid & 2 months & Yes \\
5 & Lymphoma & MRSA from pleural fluid & 2 months & Yes \\
\hline
\end{tabular}

P218 PREDICTING PNEUMOTHORAX OUTCOME BY AIR LEAK MEASUREMENT: PILOT USING DIGITAL SUCTION DEVICE

RJ Hallifax, JP Corcoran, NM Rahman; Oxford Centre for Respiratory Medicine, Oxford, UK

10.1136/thoraxjnl-2013-204457.370 
Introduction Spontaneous pneumothorax (SP) is common, with an estimated incidence of 5,000 per year in the UK. Primary spontaneous pneumothorax (PSP) can be managed with needle aspiration initially but those who fail to re-expand, and the majority of secondary SP (SSP), require admission to hospital, insertion of a chest drain and connexion to a bulky underwater drainage system. Patients with SSP have greater co-morbidities; have a long hospital stay (14-16 days) and a mortality of $\sim 16 \%$. There are no good predictors of outcome for patients with pneumothorax: those who will respond to drainage, those who have persistent air leak and those who will require surgery. Significant early air leak could be indicative of those patients who will not resolve spontaneously and will require surgery.

Aim To determine whether air leak measurement can predict patient outcome (surgical referral rate).

Methods Between December 2012 and June 2013, patients with pneumothorax managed on the Respiratory Ward of a tertiary referral centre had their "air leak" measured using a digital suction device (Thopaz, Medela UK).

Results A total of nine patients (6 SSP, 2 PSP, and 1 iatrogenic) were investigated. Air leak was measured during their in-patient stay: median of 3.5 days after admission (range 1-16 days). Three (33\%) were referred for surgical intervention for continued air leak and pneumothorax: their average air leak was $504 \mathrm{ml} / \mathrm{min}$ (range $222-952 \mathrm{ml} / \mathrm{min}$ ) compared to $77 \mathrm{ml} / \mathrm{min}$ (range $1-225 \mathrm{ml} / \mathrm{min}$ ) for the six patients not referred. The $427 \mathrm{ml} / \mathrm{min}$ difference was not statistically significant $(\mathrm{p}=0.2)$ with this sample size.

Conclusion At present, there are no good predictors of outcome for patients with pneumothorax. Air leak measurement postdrain insertion may be useful surrogate marker for on-going leak and hence non-resolving pneumothorax. This pilot study showed a difference in early leak measurement between those patients who were ultimately referred for surgery and those spontaneously resolving, and further larger studies are now warranted, comparing air leak to clinically important outcomes in pneumothorax.

\section{P219 EVALUATING MEDICAL THORACOSCOPY SERVICES AT A DISTRICT GENERAL HOSPITAL OVER THE PAST 13 YEARS}

${ }^{1} \mathrm{LM}$ George, ${ }^{2} \mathrm{HJ}$ Roberts, 'NJ Downer, 'GM Cox, ${ }^{1} \mathrm{NJ}$ Ali, ${ }^{1} \mathrm{ME}$ Roberts; 'Kingsmill Hospital, Sherwood Forest Hospitals NHS Trust, Mansfield, United KIngdom; ${ }^{2}$ Nottingham University Hospitals NHS Trust, Nottingham, United Kingdom

\subsection{6/thoraxjn-2013-204457.371}

Introduction Medical thoracoscopy is an invaluable tool in the management of undiagnosed and known malignant pleural effusions. In the UK, increasing numbers of centres are offering the procedure We present the experience from a single district general hospital that has been offering medical thoracoscopy over the last 13 years.

Methodology A retrospective analysis of 411 case notes of patients who had a medical thoracoscopy between July 2000 and April 2013.

Results 291 patients (71\%) were male. The median age was 70 years (24 to 92). In 321 patients (78\%) the procedure was done for diagnostic purposes and the procedure was done on the right side $(20 \%)$ had lung cancer, 94 (23\%) had mesothelioma and 66 ( 16\%) had other cancer.

In the 231 patients that had operator observations as appearing malignant $198(86 \%)$ were confirmed on histology. 34 ( $30 \%$ ) patients had malignancy in operator observations that

\section{Diagnostic rates}

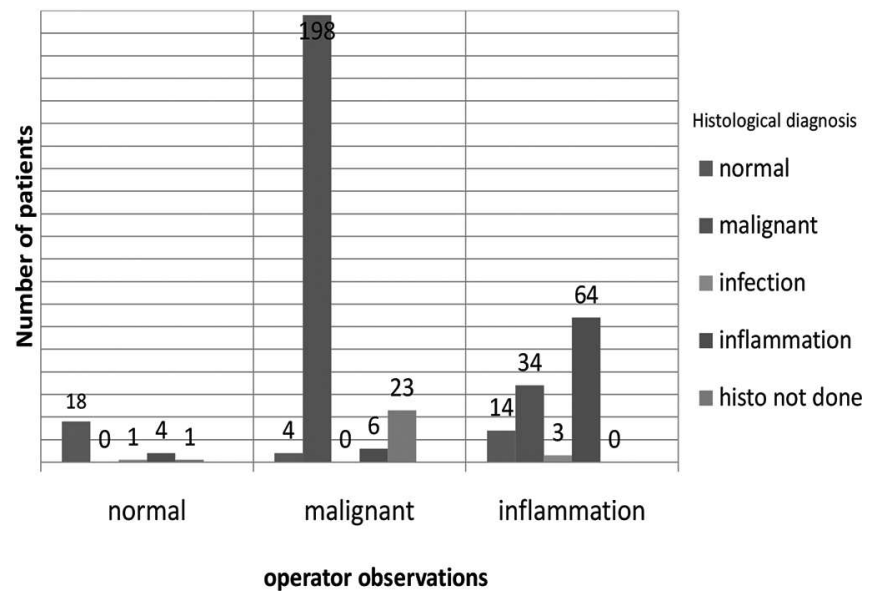

Abstract P219 Figure 1.

were reported as inflammation but reassuringly no patients had malignancy in the operator observations that were reported as appearing normal. Talc poudrage was done in 261 ( 64\%) patients. 53 patients (13\%) required a further pleural intervention within 12 months. Complications included empyema in 3 $(0.7 \%)$, renal failure in $6(1.4 \%)$ and trapped lung in $20(5 \%)$ patients. 6 patients $(1.4 \%)$ died within 15 days of the procedure and major contributors to mortality were hospital acquired infection.

Conclusions Medical thoracoscopy is an effective procedure for diagnosing and managing pleural effusions. It is associated with a low complication rate and can be performed in patients with poor performance status. Caution should be exercised in patients with multiple comorbidities and careful management of cardiovascular status post-operatively could help reduce post-procedure kidney injury.

\section{P220 EVOLVING ROLE OF THE RESPIRATORY SPECIALIST: PLEURAL ULTRASOUND SERVICE 4 YEARS ON}

B Khan, H Aminy Raouf, M Mushtaq; Darent Valley Hospital, Dartford, UK

\subsection{6/thoraxjnl-2013-204457.372}

Background The timely and safe investigation, intervention and management of pleural effusions remains discrepant with varying practices. Following the National Patient Safety Agency 2008 report highlighting 12 deaths from intercostal chest drain, the BTS recommended using ultrasound guidance when inserting chest drains. However, conventionally diagnostic and therapeutic thoracentesis have routinely been performed with either no image guidance or an "X mark the spot" in the radiology department and then transferred back to the ward for the actual procedure. All of these give rise to concern and possibly impact upon quality of care and patient safety.

Methods A retrospective analysis of $\sim 4$ years experience of providing a Respiratory team delivered ultrasound pleural service; both "inpatient" and "ambulatory".

Results In the 12 months prior to the establishment the pleural service, the radiology department did a total of 96 ultrasounds, of which 46 were " $X$ " marks the spot.

Since May 2010, 581 pleural ultrasounds have been performed; with only 5 dry taps and 3 clinically insignificant iatrogenic pneumothoraces. $41 \%$ were therapeutic thoracentesis, 